\title{
The design of reliability-optimized structure of communication system based on optical fiber ring network structure
}

\author{
Jian-Hong Yang ${ }^{1, a}$, Ning He ${ }^{1, b}$, Hao Yang ${ }^{1}$ and Jun Peng ${ }^{2, c}$ \\ ${ }^{1}$ Research and Development Center, The Second Research Institute of CAAC, Chengdu \\ P.R., No. 17, South Section 2,2nd Ring Road, China \\ ${ }^{2}$ Sichuan university, Chengdu P.R., Wangjiang Road no. 29, China \\ E-mail: ${ }^{a}$ jasongm@163.com, ${ }^{b} 441965142 @$ @q.com, ${ }^{c}$ junhongpy2@126.com \\ www.caacsri.com \\ www.scu.edu.cn
}

\begin{abstract}
When optical fiber is employed as a medium to transmit data in ring network typology structure communication system, several mal-functional work nodes in the communication system will result in malfunction in communication and loss of data, and will eventually affect performance efficiency. This paper introduces a reliability-optimized structure design which will guarantee the communication between normal work nodes and mal-functional ones and will considerably enhance the reliability of ring network communication system.
\end{abstract}

Keywords: Optical Fiber; Communication System; Ring Network; Reliability.

\section{Introduction}

Electrical signals (or optical fiber signals) information-transmission system is referred to as communication system. Communication system is an organic whole consisting of some interactive and interdependent units with specific functions and unified goal [1].

\section{The Traditional Optical Fiber Ring Network Structure}

Topological network refers to the physical connection network consisting of computer or other equipment and transmitting media by way of nodes and lines. There are two types of network node: one is transfer node which is responsible for the exchange of information, including node switches, hubs and terminal controller and so on; the other is access node, including host computer and terminals and so on. Lines refer to a variety of transmission media, including tangible and intangible ones. Optical fiber is the medium employed in this newly designed reliability-optimized structure. 
A ring network structure is established by several work nodes which in the same network is connected end to end to form a closed loop. This structure forms a loop connection of public transmitting cable in which data is transmitted along one direction from node 1 to node 7 .Such networks are usually called "token ring" [3], as is seen in Figure 1.

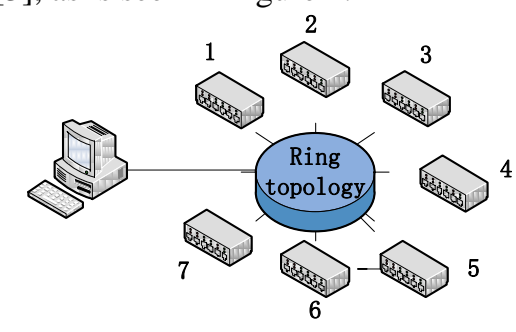

Fig. 1 Ring topology

\section{Problems of Traditional Optical Fiber Ring Network, and Reasons}

\subsection{Problems}

A ring network communication system is formed by connecting multiple processing units end-to-end using optical fiber according to topology structure ${ }^{[2]}$, as in Figure 1. When the system runs, data are transmitted subsequently from unit 1 to unit 7, and finally back to controlling computer. When unit 2 and unit 5 shut down, the entire ring communication system is open circuit; processing unit 1 cannot transmit data to host computer consistently along the path from 1 to 0 via the network, but directly transmit the data back to host computer. Nor can unit 3 and unit 4 transmit data to host computer by way of the network, causing the loss of their data.

\subsection{Reason analysis}

Node equipment suitable for setting up ring communication often has two communication ports $\mathrm{A}$ and $\mathrm{B}$, respectively standing for input and output, whose connecting method is shown in Figure 2.

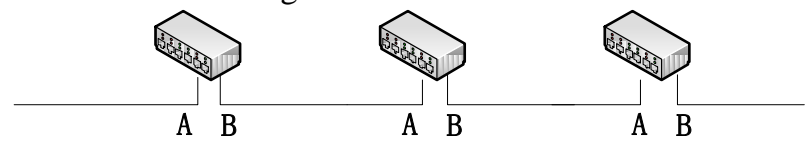

Fig. 2 Connection method

In the condition that every node works, data is input into port $\mathrm{A}$ and output from port $\mathrm{B}$. When node equipment shut down, data input and output cannot be 
realized simultaneously, disrupting communication chain and causing loss of data. In order to improve communication reliability of traditional ring network, the structure of ring network communication needs to be optimized, ensuring the functioning of input and output in case of shutdown of node or nodes.

\subsection{Reliability optimization solution}

To improve reliability of ring network communication, what comes first is to add one redundant link to the original communication link ${ }^{[4]}$. If one link ceases to work, redundant link is available for data transmission, guaranteeing the reliability of communication. The failure of one link is caused by the fault of processing unit itself, so we should find a way to make other data circumvent smoothly the mal-functioning processing unit(s) in the network without affecting subsequent communication ${ }^{[5]}$.

In accordance with the communication failure resulted from the mal-functional node equipment, it is important to optimize node equipment. The idea of the reliability-optimized structure design is to build a redundant communication link into every nodes so that when the original communication link stops working, redundant communication link automatically takes its place.

Figure 3 shows the structure of traditional optical fiber node equipment.

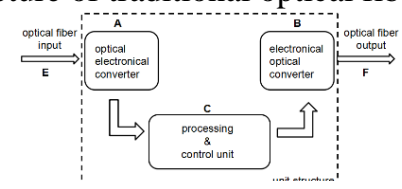

Fig. 3 Node structure before optimization

Traditional node equipment consists of 3 parts: 1) optical-electronic convert module marked as A; 2) data-processing and controlling unit marked as B; and 3) electronic-optical convert module marked as $\mathrm{C}$. In the optical fiber ring network, optical-electronic convert module (A) converts optical signal into electronic signal and input the latter into data processing and controlling unit $(\mathrm{C})$. The processed electronic signal is then converted into optical signal by electronic-optical convert module (B) and transmitted into the ring network.

Reliability-optimized design includes three steps: 1) to add a passive optical splitter to the optical signal input of the original node equipment. Since only one optical communication link needs adding, a one-into-two optical splitter is employed, as in Figure 4; 2) to add an active optical switch to the optical signal input port of original node equipment, as in Figure 5, to choose signal communication path. When active optical switch powers on, data transmission choose original communication link whereas redundant communication link is 
chosen when problem arises with the processing unit; 3) to add a controlling line between the core processing unit of original node equipment and active optical switch so that when original node equipment functions, power is supplied through the controlling line, enabling optical switch to power on; when original node equipment shuts down and fails to supply power, optical switch is power off.

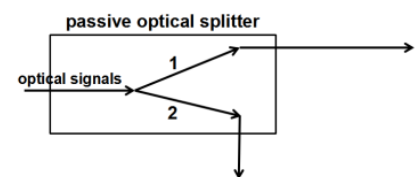

Fig. 4 Passive optical splitter working schematic

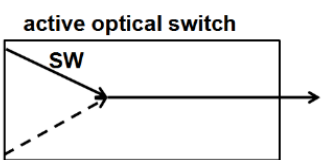

Fig. 5 Active optical switch working schematic

The following are detailed procedures of each step:

Step 1: Add passive optical splitter to the original node equipment. When optical signal enters node equipment through the splitter, the latter split the received optical signal into two branches, one connected directly to optical switch, the other connected to optical-electronic converter, ready for data processing within the node equipment.

Step 2: Active optical switch has two optical signal input ports, namely A and B, as is shown in Figure 7. The default of optical switch is that when it is not driven by power, or when it is not working, the switch is in the position of $\mathrm{A}$ and can be used to receive optical signal output from optical splitter. Input B of optical switch, however, is connected to optical signal input by electronic-optical convert module.

Step 3: When original node equipment functions, the controlling line which is connected to it output voltage, enabling active optical switch to operate; when original node equipment shut down, there is no voltage output in the controlling line so that active optical switch stops working. The structure of the optimized node is shown in Figure 6.

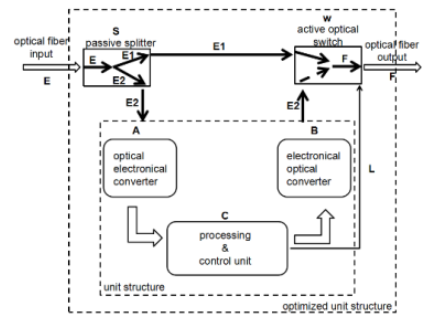

Fig. 6 Optimized node structure diagram 


\section{Test Results}

After optimization, node equipment structure has undergone a series of test. Ten pre-optimized node equipments and ten optimized ones are employed for the tests. Figure 7 is authentic scene for the tests.

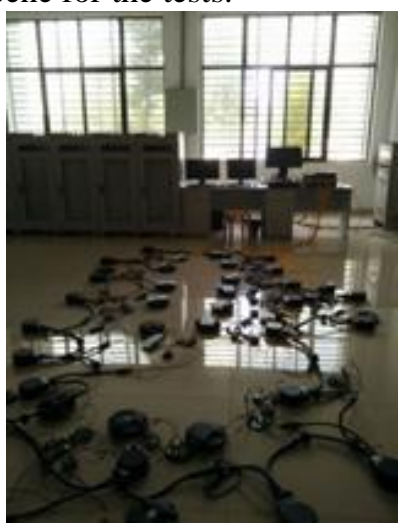

Fig. 7 Test scene

The ten pre-optimized node equipment and ten optimized ones are respectively tandem connected in the ring network, all node equipments being labeled in numerals subsequently from 1 to 9 , and 0 . A code is added to the controlling program of each equipment whose function is to output the correspondent number of the concerned node equipment when that node equipment maintains normal communication and no output in case of communication failure.

To highlight the reliability of the optimized ring network communication, the test steps are as follows:

Ten pre-optimized node equipments are tandem connected in the ring network.

1) Make the ten node equipments operate, and check the output on the computer. If the output is " 1234567890 ", then the test is done and is ready for the next step.

2) Ruin deliberately node equipment 3 and check the output on the computer. If the output is " 124567890 ", the test is done and is ready for the next step.

3) Ruin deliberately node equipments 3 and 6 , and check the output. If the output is " 127890 ", the test is done and is ready for the next step.

4) Ruin deliberately node equipments 2 and 9, and check the output. If the output is " 10 ", the test is done and is ready for the next step. 
Second, ten optimized node equipments are tandem connected in the ring network.

5) Make the ten node equipments operate, and check the output on the computer. If the output is " 1234567890 ", then the test is done and is ready for the next step.

6) Ruin deliberately node equipment 3 and check the output on the computer. If the output is " 124567890 ", the test is done and is ready for the next step.

7) Ruin deliberately node equipments 3 and 6 , and check the output. If the output is " 12457890 ", the test is done and is ready for the next step.

8) Ruin deliberately node equipments 3,6 and 9, and check the output. If the output is "1245780", the test is done and is ready for the next step.

9) Ruin deliberately node equipments 2 and 9, and check the output. If the output is " 13456780 ", the test is done and is ready for the next step.

10) Ruin deliberately node equipments 1 and 0 , and check the output. If the output is " 23456789 ", the test is done and is ready for the next step.

The detailed test results are indicated in Table 1.

Tab. 1 Test results

\begin{tabular}{|c|c|c|c|}
\hline $\begin{array}{l}\text { Test serial } \\
\text { number }\end{array}$ & Test contents & Output & results \\
\hline \multicolumn{4}{|c|}{10 pre-optimized nodes are tandem connected to set up a ring network } \\
\hline 1 & 10 test nodes work properly & 1234567890 & Correct \\
\hline 2 & NO.3 test node is ruined deliberately & 124567890 & Correct \\
\hline 3 & NO.3 and NO.6 test nodes are ruined deliberately & 127890 & Correct \\
\hline 4 & NO.2and NO.9 test nodes are ruined deliberately & 10 & Correct \\
\hline \multicolumn{4}{|c|}{10 optimized nodes are tandem connected to set up a ring network } \\
\hline 5 & 10 test nodes work properly & 1234567890 & Correct \\
\hline 6 & NO. 3 test node is ruined deliberately & 124567890 & Correct \\
\hline 7 & NO.3 and NO.6 test nodes are ruined deliberately & 12457890 & Correct \\
\hline 8 & $\begin{array}{c}\text { NO.3 and NO.6 and NO.9 test nodes are ruined } \\
\text { deliberately }\end{array}$ & 1245780 & Correct \\
\hline 9 & NO.2and NO.9 test nodes are ruined deliberately & 13456780 & Correct \\
\hline 10 & NO.1 and NO.0 test nodes are ruined deliberately & 23456789 & Correct \\
\hline
\end{tabular}

As is shown in Table 1, with the optimization of the structure of node equipments, the reliability of the entire ring communication system has been 
greatly improved and has satisfactorily solved the communication problem with the current ring network.

\section{Conclusion}

In conclusion, the newly designed reliability-optimized structure introduced in this paper guarantees the communication between normal work nodes and mal-functional ones and will considerably enhance the reliability of ring network communication system.

This highly reliable communication method is based on the optical fiber ring network structure and is designed and tested in the process of fulfilling a series of project. The reliability of communication can be greatly improved by adding a passive optical splitter and an active optical-electronic switch to all node equipments in the ring network. Though there is modest increase in the cost of the ring network, its contribution to high reliability of communication outweighs. So this reliability-optimized structure design is of great practical value and significance to projects demanding high communication reliability.

\section{References}

1. J. G. Proakis, Digital Communications 4th ed.(Beijing, China, 2001).

2. Groth and David, Network+ Study Guide4th ed. Sybex, Inc. ISBN 0-7821-4406-3.

3. Shan Yanhu and Zhen Guoyong (eds.), An LVDS bus protocol design and implementation based on the token ring, Electrical Measurement \& Instrumentation,Vol. 47,Num.3,2010,pp.77-80. (In Chinese).

4. Wang Zhenxi, Reliability, redundancy and fault-tolerant technology. (Beijing, China, 1991). (In Chinese).

5. Elsayed.A.Elsayed, Reliability Engineering2nd ed. (Beijing, China, 2013). (In Chinese). 